\section{Professor Anthony Clare}

Dear Editor - I was most saddened to hear about the death of Professor Anthony Clare. Like innumerable people he was extremely helpful to me in many ways since 1973 when he introduced me to the Maudsley Hospital in London, where I was shortly after to work. Since then I found him unfailingly helpful when I approached him about various academic matters. His contribution to the reducing the stigma of psychiatry worldwide has been enormous. I know of no other psychiatrist who has achieved so much in this area in the 20th century. He will be much missed by everyone.

Michael Fitzgerald Henry Marsh Professor Child and Adolescent Psychiatry, Trinity College Dublin, Dublin 2, Ireland

\section{Intra-arterial injection of zolpidem and substance misusers}

Dear Editors - Hypnotics have long been drugs of abuse by both substance misusers and patients attending adult psychiatric and general practitioner clinics. Zolpidem is licensed for the short term treatment of insomnia. However, we are seeing an increasing amount of abuse of zolpidem particularly among intravenous drug users (IDU).

Zolpidem abusers can take the drug orally, intravenously or by crushing or snorting it. Zolpidem abuse can occur in those who use or are prescribed it for longer than is recommended (four to six weeks) and at doses higher than $10 \mathrm{mg}-20 \mathrm{mg}$. Its affects are also enhanced when used with other substances such as alcohol. Dependence and withdrawal is commonly seen in chronic users of the drug. ${ }^{3} \mathrm{~A}$ case report detailed the history of a lady who experienced epileptic seizures following abrupt withdrawal of zolpidem following a history of chronic use at high doses. ${ }^{1}$ Other reported side effects include hallucinations, delusions, ataxia, euphoria, impulsivity and amnesia. Intravenous abuse is particularly hazardous as doses as small as $5 \mathrm{mg}$ can produce serious effects.

In the Drug Treatment Centre and Advisory Board we have more recently seen one serious consequence associated with injection of zolpidem by intravenous drug users (IDU). Two patients who regularly injected zolpidem developed 'arterial microspasm' and this resulted in 'tissue necrosis and the possibility of loss of large areas of skin and digits' according to their consultant vascular surgeon.

Both patients had a history of intravenous drug use and had injected zolpidem a number of times previously. On one occasion within hours of injecting both patients complained of severe pain, difficulty weight bearing and a noticeable change in colour in the skin in one of their feet. One patient reported that they boiled two tablets of zolpidem and injected it into their foot, while the other patient reported crushing two tablets and injecting into their groin. They denied injecting other illicit substances at the time.

Both patients presented to hospital within hours of injecting and the onset of symptoms. Duplex scanning revealed 'severe microvascular spasm secondary to intra-arterial injec- tion of zolpidem'. One patient required emergency treatment with intravenous (IV) heparin to prevent irreversible tissue destruction and loss of digits. The other patient was treated with an anticoagulant infusion and tinzaparin sodium (Innohep) and was given further anticoagulant treatment for a month following discharge.

There have been a limited number of papers suggesting an association between zolpidem and tissue ischaemia. One published paper reported that microcrystalline cellulose, an ingredient in zolpidem, acts as a potent embolic agent resulting in microvascular embolisation and ischaemia. ${ }^{2}$

These cases serve to highlight the dangers of intravenous (IV) substance misuse. We regularly inform patients about the risks of injecting illicit substances amongst which include overdose, infective diseases, abscesses, deep venous thrombosis and infective endocarditis. However, we should now highlight the dangers of injecting zolpidem as even a relatively small dose can produce serious consequences. We would like to invite your readers for their comment on similar cases they may have come across within their clinical practice.

\section{Marie Whitty and John O Connor Drug Treatment Centre and Advisory Board Trinity Court, 30-32 Pearse Street, Dublin 2, Ireland.}

\section{References}

1. Aragona M. Abuse, dependence, and epileptic seizures after zolpidem withdrawal: review and case report. Clin Neuropharmacol 2000; 23: 281-3.

2. Chang MY et al. Irreversible ischemic hand following intraarterial injection of zolpidem powder. J Toxicol: Clin Toxicol 2003: 41: 1025-1028.

3. Madrak LN, Rosenberg MSN. Zolpidem Abuse, Letter to the Editor. Am J Psychiatry 2001; 158: 1330-1331.

\section{Caffeine and suicide}

Dear Editor - In August 2007, the BBC reported the hospitalisation of a 17 year old due to accidental overdose 1 in the UK. A 17 year old taking an overdose in the UK is hardly unusual so what was so interesting about this case that it sparked the interest of the national media? The reason was that the offending substance was the world's most commonly consumed drug: caffeine. The teenager in question had drunk seven double measures (thinking they were singles) of espresso coffee during a single work-shift and was admitted due to the toxic effects of caffeine. In this instance, the overdose was neither intentional nor fatal but the case illustrates that, despite it ubiquity, caffeine is a chemical not without its dangers.

Indeed, this was not the first time that the attention of the British media was enthused by a caffeine overdose. In January 2002, a 20 year chemistry student committed suicide in Wales after consuming almost four hundred tablets of Pro Plus, $^{2}$ a caffeine-containing stimulant popular among students. The coroner's verdict confirmed the cause of death as suicide by caffeine overdose. Later that same year, the UK press reported the suicide of a 58 year woman who overdosed on several hundred Pro Plus tablets which she consumed with cans of the caffeine-containing energy drink Red Bull. ${ }^{3}$ Her blood caffeine levels were found to $776 \mathrm{mg} /$; the fatal level of blood caffeine is estimated at around 